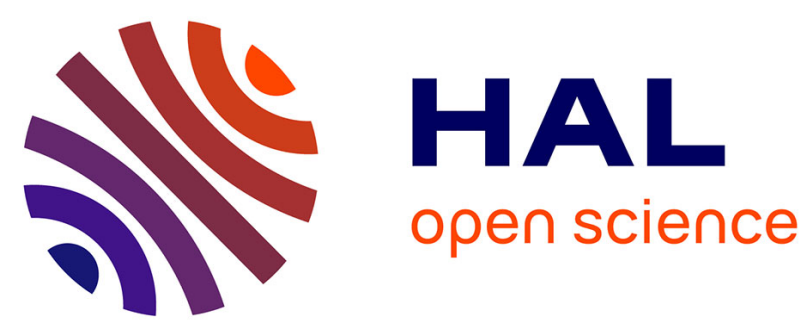

\title{
Guided wave phase velocity measurement using multi-emitter and multi-receiver arrays in the axial transmission configuration
}

Jean-Gabriel Minonzio, Marilyne Talmant, Pascal Laugier

\section{- To cite this version:}

Jean-Gabriel Minonzio, Marilyne Talmant, Pascal Laugier. Guided wave phase velocity measurement using multi-emitter and multi-receiver arrays in the axial transmission configuration. Journal of the Acoustical Society of America, 2010, 127 (5), pp.2913 - 2913. 10.1121/1.3377085 . hal-01394310

\section{HAL Id: hal-01394310 \\ https: / hal.sorbonne-universite.fr/hal-01394310}

Submitted on 9 Nov 2016

HAL is a multi-disciplinary open access archive for the deposit and dissemination of scientific research documents, whether they are published or not. The documents may come from teaching and research institutions in France or abroad, or from public or private research centers.
L'archive ouverte pluridisciplinaire HAL, est destinée au dépôt et à la diffusion de documents scientifiques de niveau recherche, publiés ou non, émanant des établissements d'enseignement et de recherche français ou étrangers, des laboratoires publics ou privés. 


\section{Guided wave phase velocity measurement using multi-emitter and} multi-receiver arrays in the axial transmission configuration

Jean-Gabriel Minonzio a), Marilyne Talmant, Pascal Laugier

UPMC Univ Paris 06, UMR 7623, LIP,

15 rue de l'école de médecine F-75005, Paris, France

a) electronic mail: jean-gabriel.minonzio@upmc.fr

running title: guided wave velocity measurement using arrays in contact

30 June 2009

5 March 2010 


\section{Abstract}

This paper is devoted to a method of extraction of guided waves phase velocities from experimental signals. Measurements are performed using an axial transmission device consisting of a linear arrangement of emitters and receivers placed on the surface of the inspected specimen. The technique takes benefit of using both multiple emitters and receivers and is validated on a reference wave guide. The guided mode phase velocities are obtained using a projection in the singular vectors basis. The singular vectors are determined by the singular values decomposition (SVD) of the response matrix between the two arrays in the frequency domain. This technique enables to recover accurately guided wave phase velocity dispersion curves. The SVD based approach was designed to overcome limitations of spatiotemporal Fourier transform for receiver array of limited spatial extent as in the case of clinical assessment of cortical bone in axial transmission.

PACS numbers

4320Ye Measurement methods and instrumentation, 4380Vj Acoustical medical instrumentation and measurement techniques 4320Mv Waveguides, wave propagation in tubes and ducts 4360Fg Acoustic array systems and processing, beam-forming 


\section{INTRODUCTION}

Different quantitative ultrasound (QUS) techniques are currently developed for clinical assessment of human bone status. ${ }^{1-3}$ The goal is to determine ultrasound-based indicators of bone strength, suitable for the discrimination of osteoporotic patients from healthy subjects. ${ }^{4}$ Alternatively, the possibility of bone properties determination from ultrasound measurements, for instance cortical thickness or elasticity, has been explored. ${ }^{5-7}$ One of the most promising recent development in this field is the so called "axial transmission" technique: a set of emitter(s) and receiver(s) are linearly arranged on the same side of a skeletal site, for instance the forearm or the leg. ${ }^{8-10}$ The signals obtained at the receiver(s) are the combination of all waves propagating axially along the long axis of the bone. A few studies indicate that cortical bones support guided waves propagation, despite absorption and heterogeneity in geometry and elasticity. ${ }^{11}$

In one development of the axial transmission technique, attention was focused in determining in the time domain the velocity of the first arriving signal, denoted FAS. ${ }^{12,13}$ The FAS can be interpreted as a guided $S_{0}$ wave in the low frequency regime (i.e., low cortical thickness-towavelength ratio) and as a lateral compression wave in the high frequency regime (i.e., high cortical thickness-to-wavelength ratio). ${ }^{14}$ Alternatively, other studies have focused attention on the most energetic contribution arriving after the FAS. Signal processing techniques were proposed in vitro to isolate this signal component and to measure its phase velocity: group velocity filtering technique prior to spatio-temporal Fourier transform ${ }^{15,16}$ or the singular value decomposition in the space-time domain. ${ }^{17,18}$ In in vitro measurements, this contribution is identified with the $A_{0}$ plate guided mode, or its counterpart for the tube model, the $F(1,1)$ flexural mode.

Moreover, higher order guided modes have been experimentally identified on in vitro animal bones. Different techniques were used to extract guided mode phase or group 
velocities. The $L(0, n)$ longitudinal tube wave phase velocities $(1 \leq n \leq 3)$ were measured on a bovine tibia. ${ }^{19}$ Authors used angled beam transducers and analyzed the received signals with the phase spectrum method. ${ }^{20}$ The method required a minimal distance between the emitter and receiver of about $70 \mathrm{~mm}$ and a scanning length of about $80 \mathrm{~mm}$. On a sheep tibia, guided waves were also experimentally observed. ${ }^{21}$ Authors used two fixed transducers and obtained group velocities using time-frequency analysis. ${ }^{22}$ Results were interpreted as Rayleigh-Lamb guided modes, $A_{n}$ and $S_{n}(0 \leq n \leq 2)$. However an accurate model is required to interpret the results.

The clinical relevance of ultrasound velocities to reflect specific aspects of bone strength such as cortical thickness, stiffness, porosity is partly established. It was found in several clinical studies that the FAS velocity discriminates healthy subjects from osteoporotic patients. ${ }^{23-25}$ In addition, a FAS velocity database, measured in vivo, was used in a technique of elasticity identification. ${ }^{7}$ From $A_{0}$ phase velocity data, inversion schemes were proposed to estimate cortical thickness. ${ }^{5}$ However, the $A_{0}$ guided wave presents a strong coupling with the surrounding soft tissues which reduces its sensitivity to bone properties in clinical measurement. $^{26}$

Currently, there is no clinical measurement of higher order guided waves. There is a need to implement a robust method to extract guided mode phase velocities adapted to clinical requirements. Particularly, as the probe dimensions are limited by the accessibility to the skeletal site or bone and soft tissue, classing signal processing methods ${ }^{16,18,20,22}$ can not be directly applied. Thus, the aim of this paper is to introduce a method of extraction of guided mode phase velocities from in vivo signals. Our approach is based on the singular value decomposition (SVD) applied to the configuration of multi-emitter and multi-receiver arrays in axial transmission geometry. SVD is a widely used filtering tool. The interpretation of the 
singular vectors and singular values is adapted and specific to each case and array configuration. $^{27-29}$

The paper is organized as follows. First, the principle of the method is presented in Sec. II: the phase velocities are measured using the singular vectors of the response matrix, interpreted as the basis of the received signal subspace. The advantages of the method are then described: the signal and noise separation and the improvement of the ability to evaluate phase velocities. To this end, two specific thresholds are discussed. In Sec. III the accuracy and effectiveness of the method are tested on experimental measurements on a reference wave guide. Two probes of central frequency equal to 1 and $2 \mathrm{MHz}$, developed for the axial transmission approach, are used. ${ }^{13}$ The experimental set up, the evaluation domain and the resolution are then discussed. Finally experimental phase velocities arecompared with theoretical ones and with those obtained with the spatio-temporal Fourier transform.

\section{THEORY}

\section{A. Response matrix}

A single probe consisting of two separated arrays are used in contact with a metallic plate, using coupling gel (Fig. 1). Superscripts E and R refer respectively to the emission and reception arrays. Thus the emission array contains $N^{\mathrm{E}}$ emitters of position $x_{i}^{\mathrm{E}}$, with $1 \leq i \leq N^{\mathrm{E}}$. Likewise, the reception array contains $N^{\mathrm{R}}$ receivers of position $x_{j}^{\mathrm{R}}$, with $1 \leq j \leq N^{\mathrm{R}}$. 


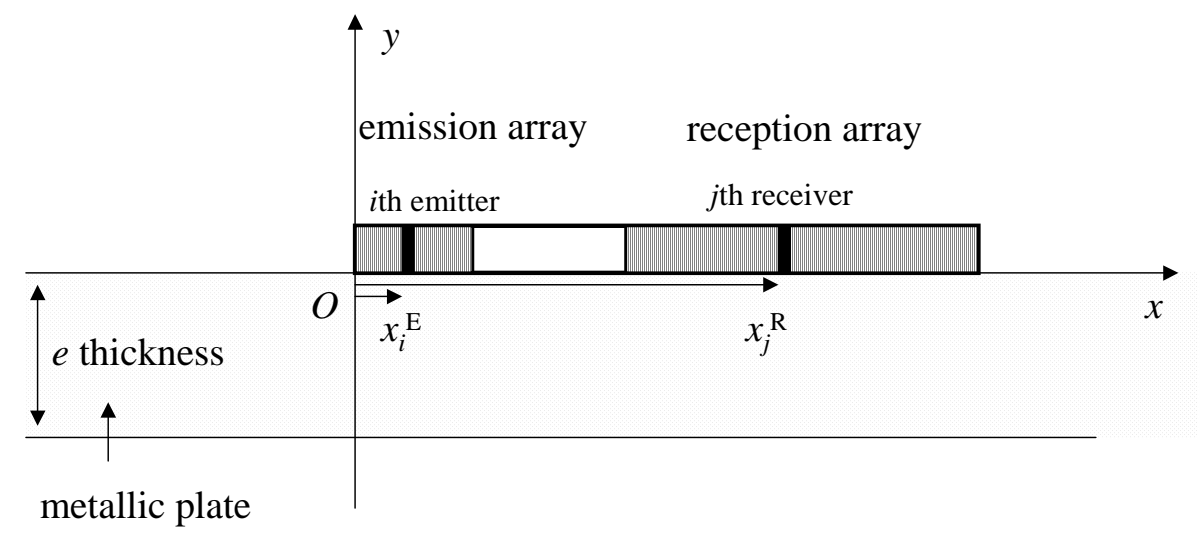

Fig. 1. Geometry of the problem: the emission and reception arrays are in contact with the metallic plate.

The response to a pulse emitted by the ith emitter, received by the $j$ th receiver after propagation in the medium, is denoted $h_{j i}(t)$. It corresponds to the impulse response between the two transducers. The number of responses is equal to $N^{\mathrm{R}}$ multiplied by $N^{\mathrm{E}}$. The received signal $r_{j i}(t)$ on the $j$ th receiver is given by the sum of the convolutions of the emissions $e_{j i}(t)$ with the impulse responses $h_{j i}(t)$ as

$$
r_{j i}(t)=\sum_{i=1}^{N^{\mathrm{E}}} h_{j i}(t) \otimes e_{i i}(t) .
$$

The temporal Fourier transform of Eq. (1) writes

$$
R_{j i}(f)=\sum_{i=1}^{N^{\mathrm{E}}} H_{j i}(f) E_{i i}(\omega),
$$

with $f$ the frequency and $R_{j}(f), H_{j i}(f), E_{i}(f)$ the temporal Fourier transforms of $r_{j}(t), h_{j i}(t), e_{i}(t)$ respectively. The $N^{\mathrm{R}} \times N^{\mathrm{E}}$ matrix $\mathbf{R}$ contains the $N^{\mathrm{R}} \times N^{\mathrm{E}}$ Fourier transforms $R_{j i}(f)$. Likewise, the $N^{\mathrm{E}} \times N^{\mathrm{E}}$ diagonal matrix $\mathbf{E}$ contains the $N^{\mathrm{E}}$ Fourier transforms $E_{i i}(f)$. The relation between the two vectors $\mathbf{R}$ and $\mathbf{E}$ writes, at a given frequency $f$

$$
\mathbf{R}=\mathbf{H} . \mathbf{E},
$$


with $\mathbf{H}$ the $N^{\mathrm{R}} \times N^{\mathrm{E}}$ matrix containing the elements $H_{j i}(f)$ equal to the Fourier transforms of the impulse responses $h_{j i}(t)$. The $\mathbf{R}$ matrix, called afterward "response matrix", is characteristic of the “emission -propagation medium - reception” system (Fig. 1).

\section{B. Singular vectors basis}

The Singular Value Decomposition (SVD) of the response matrix $\mathbf{R}$ writes

$$
\mathbf{R}=\sum_{n=1}^{N^{\mathrm{E}}} \mathbf{U}_{n} \sigma_{n}{ }^{t} \mathbf{V}_{n}^{*},
$$

where the notations ${ }^{t}$ and ${ }^{*}$ denote the transpose and conjugation operations. The number of experimental singular values $\sigma_{n}$ is equal to minimum size of the arrays, i.e. the minimum between $N^{\mathrm{E}}$ and $N^{\mathrm{R}}$, equal in the following to $N^{\mathrm{E}}$. The notation $\mathbf{V}_{n}$ refers to an emission singular vector, of dimension $N^{\mathrm{E}} \times 1$, and $\mathbf{U}_{n}$ to a reception singular vector, of dimension $N^{\mathrm{R}} \times 1$. These two vectors are associated with the singular value $\sigma_{n}$. The reception singular vectors $\mathbf{U}_{n}$ are the eigenvectors of $\mathbf{R} .{ }^{t} \mathbf{R}^{*}$, whereas, the emission vectors $\mathbf{V}_{n}$ are the eigenvectors of ${ }^{t} \mathbf{R}^{*}$. R. In both cases the eigenvalues are the square of the singular values $\sigma_{n}{ }^{30}$ In the following, the singular values are considered as sorted in a decreasing order, i.e. $\sigma_{1}>\sigma_{2}>\ldots$

The singular vectors $\mathbf{U}_{n}\left(\right.$ resp. $\mathbf{V}_{n}$ ) are normalized and define an orthogonal basis of the received (resp. emitted) signals. In particular any spatial plane wave, propagating along the reception array direction, i.e. along the $x$ axis (Fig. 1), can be expressed in the reception basis. Consider a spatial plane wave $\mathbf{e}^{p w}(f, c)$ depending on the phase velocity $c$ and the frequency $f$, defined on the $j$ th receiver as

$$
e_{j}^{p w}(f, c)=\frac{1}{\sqrt{N^{\mathrm{R}}}} \exp \left(\mathrm{i} \frac{2 \pi f}{c} x_{j}^{\mathrm{R}}\right),
$$


The previous vector is normalized. As the phase velocity $c$ is real, equation 5 corresponds to the propagating part of the spatial plane wave. Its expression on the basis defined by the reception singular vectors $\mathbf{U}_{n}$ is

$$
\mathbf{e}^{p w}(f, c)=\sum_{n=1}^{N^{\mathrm{E}}}\left\langle\mathbf{e}^{p w}(f, c) \mid \mathbf{U}_{n}\right\rangle \mathbf{U}_{n},
$$

with the notation $\left\langle\mathbf{e}^{p w}(f, c) \mid \mathbf{U}_{n}\right\rangle$ corresponding to the Hermitian scalar product, equal to ${ }^{t}\left[\mathbf{e}^{p w}(f, c)\right]^{*} \cdot \mathbf{U}_{n}$. If the array is placed along the (Oy) direction (Fig. 1), as for example a vertical array in shallow water guide, ${ }^{28}$ each reception singular vector $\mathbf{U}_{n}$ is associated with a single guided mode. If the array is distant from the wave guide in the DORT configuration, DORT being the French acronym for decomposition of the time-reversal operator, the singular vectors are associated with guided mode scattering directions. ${ }^{30}$ In the axial transmission geometry, as the received signals are measured along the $(O x)$ direction, a reception singular vector corresponds to a combination of guided modes as discussed in paragraph III.D.

\section{Noise and signal separation}

One of the advantages of the SVD approach is its ability to separate noise and signal subspaces. ${ }^{29-31}$ Indeed, using an intermediate order $m$ corresponding to the limit between the two subspaces, Eq. (6) can be rewritten as

$$
\mathbf{e}^{p w}(f, c)=\sum_{n=1}^{m}\left\langle\mathbf{e}^{p w}(f, c) \mid \mathbf{U}_{n}\right\rangle \mathbf{U}_{n}+\sum_{n=m+1}^{N^{\mathrm{E}}}\left\langle\mathbf{e}^{p w}(f, c) \mid \mathbf{U}_{n}\right\rangle \mathbf{U}_{n} .
$$

The order $m$ is defined at each frequency using a threshold $t_{1}$ applied to the singular values $\sigma_{n}$. On the one hand, if the singular value is larger than that the threshold $t_{1}$, the corresponding reception singular vector $\mathbf{U}_{n}$ is associated with the signal subspace. On the other hand, if the singular value is less than that the threshold $t_{1}$, the corresponding reception singular vector $\mathbf{U}_{n}$ 
is associated with noise. In the following, the signal singular vectors are retained whereas the noise singular vectors are eliminated. Thus the norm of the propagating spatial plane wave on the basis of the signal subspace becomes

$$
\left\|\mathbf{e}^{p w}(f, c)\right\|_{\left\{\mathbf{U}_{n \leq m}\right\}}=\sqrt{\sum_{n=1}^{m}\left|\left\langle\mathbf{e}^{p w}(f, c) \mid \mathbf{U}_{n}\right\rangle\right|^{2}},
$$

with the notation || used for the modulus of complex numbers.

The value of the threshold $t_{1}$ is heuristically chosen, based on an estimation of the signal-to-noise ratio. It depends on the estimated number of measurable guided modes and on the noise level at each frequency as discussed in Ref. 31. The threshold can be chosen fixed or variable with frequency and attenuation. Examples are discussed in Sec. III.

\section{Evaluation of the guided mode phase velocities}

As $m$, the dimension of the signal subspace, is less than the number of receivers $N^{\mathrm{R}}$, i.e. the dimension of the vector $\mathbf{e}^{p w}(f, c)$ [Eq. (5)], the basis defined by the signal reception singular vectors $\mathbf{U}_{n \leq m}$ is incomplete. It implies that the norm of the propagating spatial plane wave expressed on the basis of the signal subspace, i.e. $\left\|\mathbf{e}^{p w}(f, c)\right\|_{\left\{\mathbf{U}_{n \leq m}\right\}}$ [Eq. (8)], is less than 1. Consequently, each value in the $(f, c)$ domain of that norm ranges from 0 to 1 . This value reflects how the spatial plane wave is represented in the basis of the signal subspace. On the one hand, if the value is small compared to 1 , the spatial plane wave is absent of the received signals. On the other hand, if the value is close to 1 , the spatial plane wave corresponds to a experimental propagating guided mode, present in the received signals. To enhance the contrast between the low and high values, the square of the norm is used in the following. And a second threshold $t_{2}$ is then applied to the norm in order to reduce the range from $t_{2}$ to 1 . Thus a new function, denoted $\operatorname{Norm}(f, c)$, is defined as 


$$
\left\{\begin{array}{ll}
\operatorname{Norm}(f, c)=\left\|\mathbf{e}^{p w}(f, c)\right\|_{\left\{\mathbf{U}_{n \leq m}\right\}}^{2} & \text { if }\left\|\mathbf{e}^{p w}(f, c)\right\|_{\left\{\mathbf{U}_{n \leq m}\right\}}^{2} \geq t_{2} \\
\operatorname{Norm}(f, c)=0 & \text { if }\left\|\mathbf{e}^{p w}(f, c)\right\|_{\left\{\mathbf{U}_{n \leq m}\right\}}^{2}<t_{2}
\end{array} .\right.
$$

A maximum of the Norm function in the $(f, c)$ domain, larger than $t_{2}$, provides the phase velocity $c$ of a guided mode present in the signal subspace at the frequency $f$. The value of the second threshold $t_{2}$ is also heuristically chosen considering the variations of the square of the norm $\left\|\mathbf{e}^{p w}(f, c)\right\|_{\left\{\mathbf{U}_{n \leq m}\right\}}^{2}$. In the examples discussed in Sec. III the threshold $t_{2}$ is chosen equal to 0.5 .

\section{EXPERIMENTAL RESULTS AND DISCUSSION}

\section{A. Experimental set up}

Two probes developed at the LIP (Laboratoire d’Imagerie Paramétrique), for the bone characterization, are used. ${ }^{13}$ Their central frequencies $f_{0}$ are equal respectively to 1 and 2 $\mathrm{MHz}$. The number $N^{\mathrm{E}}$ of emitters is equal to $3\left(f_{0}=1 \mathrm{MHz}\right)$ and $8\left(f_{0}=2 \mathrm{MHz}\right)$. In both cases, the number of receivers $N^{\mathrm{R}}$ is equal to 14 . Thus the number of emitters, which is less than the number of receivers, corresponds to the number of experimental singular values. A coupling gel is used in order to improve the acoustical impedance matching. Emitted signals are pulses with bandwidth centred on the central frequency $f_{0}$. The reception array pitch is equal to $0.80 \mathrm{~mm}\left(f_{0}=1 \mathrm{MHz}\right)$ and $0.38 \mathrm{~mm}\left(f_{0}=2 \mathrm{MHz}\right)$.

The experimental scattering medium is a $2 \mathrm{~mm}$ thickness copper plate. The free plate guided modes are given by the Rayleigh-Lamb dispersion equation. ${ }^{32}$ No attenuation is considered in this model. The modes are numerated following their cut-off frequencies $f_{\mathrm{c} .}{ }^{32,33}$ The cut-off frequencies of the $A_{2 n+1}$ and $A_{2 n}$ modes satisfy respectively $f_{\mathrm{c}} e=(n+1 / 2) c_{\mathrm{T}}$ and $n c_{\mathrm{L}}$, with $e$ the plate thickness (Fig. 1). Likewise, the cut-off frequencies of the $S_{2 n+1}$ and $S_{2 n}$ modes 
satisfy respectively $f_{\mathrm{c}} e=(n+1 / 2) c_{\mathrm{L}}$ and $n c_{\mathrm{T}}$. These frequencies are shown in the upper scale of Fig. 4.

\section{B. Resolution and evaluation domain of the system}

The resolution is defined similarly to the spatio-temporal Fourier transform as the ability of the system to discriminate two different phase velocities. The resolution can be estimated considering an ideal case of a spatial plane wave of phase velocity $c_{n}$, denoted $\mathbf{e}^{p w}\left(f, c_{n}\right)$, with a constant amplitude along the array. In this case, the Norm function is given by the scalar product $\left|\left\langle\mathbf{e}^{p w}(f, c) \mid \mathbf{e}^{p w}\left(f, c_{n}\right)\right\rangle\right|^{2}$ equal to

$$
\operatorname{Norm}(f, c)=\left|\operatorname{sinc}\left(\pi f \frac{c_{n}-c}{c_{n} c} L^{\mathrm{R}}\right)\right|^{2},
$$

with $L^{\mathrm{R}}$ the length of the reception array. Thus, the resolution is given by the size of the main lobe of the sinc function, i.e. $f \Delta c L^{\mathrm{R}} / c^{2} \approx 1$, where $\Delta c$ is the difference between $c_{n}$ and $c$. With $\lambda$ the wavelength satisfying $\lambda=c / f$, the resolution condition writes

$$
\frac{\Delta c}{c} \geq \frac{\lambda}{L^{\mathrm{R}}}
$$

Previous equation means that two velocities are resolved when their difference $\Delta c / c$ is larger than the ratio $\lambda / L^{\mathrm{R}}$. If the amplitude of a guided mode decreases along the array, due to attenuation or geometric dispersion, the resolution function, defined by Eq. (10), will be affected in two ways: its maximum will decrease and its width will increase. Thus, the second threshold value $t_{2}$ has to be adapted to each experiment.

Furthermore the evaluation domain can be estimated comparing the wavelength $\lambda$ with two typical lengths: the size of the array $L^{\mathrm{R}}$ and the array pitch, equal to $L^{\mathrm{R}} / N^{\mathrm{R}}$. First, the Shannon criterion is verified when the half wavelength $\lambda / 2$ is larger than the array pitch. 
Secondly, the half wavelength should also be smaller than the array size. Thus the range $\frac{2}{N^{\mathrm{R}}} \leq \frac{\lambda}{L^{\mathrm{R}}} \leq 2$ corresponds to the evaluation domain, where the guided mode phase velocities $c_{n}{ }^{\text {exp }}$ can be evaluated. Figure 2 shows this domain in the frequency-phase velocity $(f, c)$ plane. Thus, each linear function passing through the origin satisfies $c=\lambda f$.

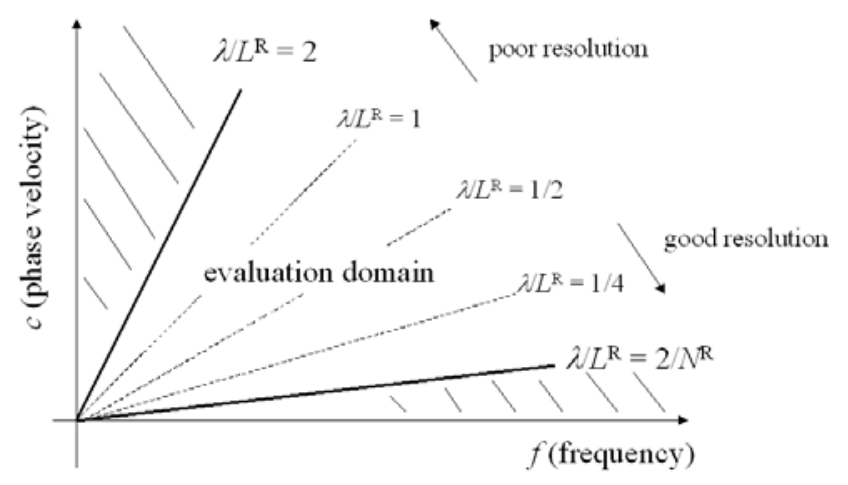

Fig. 2. The evaluation domain is shown outside the dashed domain. The resolution condition is given by Eq. (11): two velocities are resolved when their difference $\Delta c / c$ is larger than the ratio $\lambda / L^{\mathrm{R}}$ shown as linear function. The boundaries are shown on the experimental results (Fig. 4). 


\section{Experimental results: Singular values and phase velocities}

Figure 3 shows the singular values $\sigma_{n}$ as function of the frequency-thickness $f e$ product obtained with the two probes, $1 \mathrm{MHz}(a)$ and $2 \mathrm{MHz}(b)$,for a $2 \mathrm{~mm}$ thick copper plate. In both cases, the first singular value $\sigma_{1}$ is predominant at low frequency, for $f e<1 \mathrm{MHz}$.mm. It is related to the strong normal displacement associated with the first anti-symmetric $A_{0}$ mode. ${ }^{34}$ The large peaks correspond to thickness resonance of modes, associated with the cutoff frequencies, as for example the $A_{1}$ mode ( $f e$ around $\left.1.6 \mathrm{MHz} . \mathrm{mm}\right)$ and $A_{2}$ mode ( $f e$ around 5.5 MHz.mm). The thin peaks corresponds to the resonances associated with the zero group velocity $(\mathrm{ZGV})$ modes, $^{33}$ for the $S_{2}$ mode $(f e=2.10 \mathrm{MHz} . \mathrm{mm})$ and $S_{6}$ mode $(f e=$ 6.85 MHz.mm). These modes are listed on the upper scale of Fig. 4 showing the corresponding guided mode phase velocities.

The number of significant singular values ranges from 1 to 5 depending on frequency. That number corresponds to the number $m$ of significant singular vectors used in Eq. (8). It depends on the signal to noise threshold $t_{1}$, shown with a curved line. The threshold $t_{1}$ has been heuristically fixed for the two probes by searching the best experimental separation between the signal and noise subspaces. The $1 \mathrm{MHz}$ probe [Fig. 3(a)] presents a second harmonic bandwidth, for $f$ ranging from 2.5 to $4 \mathrm{MHz}$, i.e. for $f e$ ranging from 5 to 8 MHz.mm. 

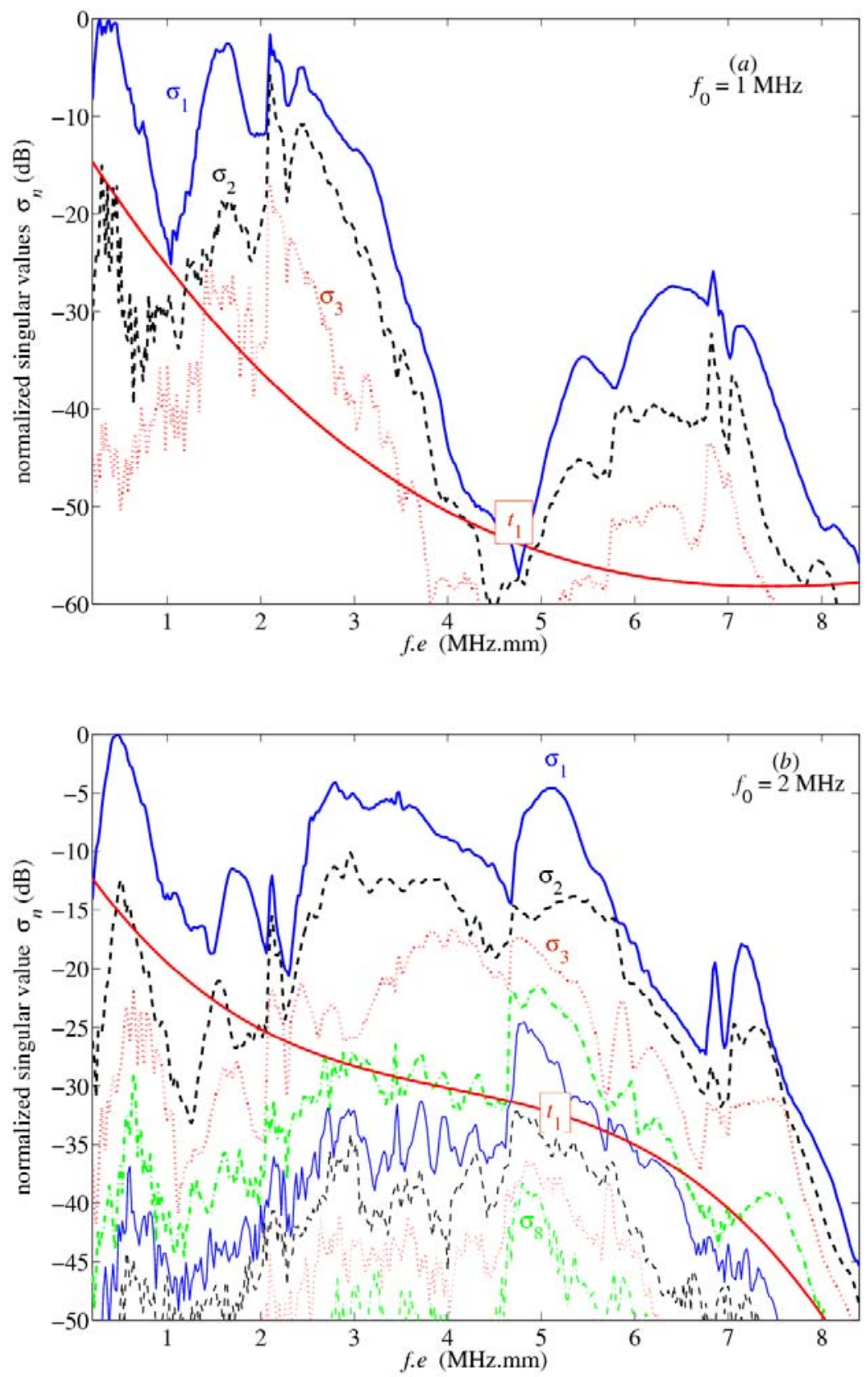

Fig. 3. (color online) Normalized singular values in $\mathrm{dB}$ scale versus frequency-thickness $f e$ (MHz.mm) for a $2 \mathrm{~mm}$ thick copper plate: the central frequency of the probe is equal to 1 $\mathrm{MHz}(a)$ and $2 \mathrm{MHz}(b)$. The number of experimental singular values (3 or 8) corresponds to the number of emitters. The signal-to-noise threshold $t_{1}$ is shown with a curved line. The singular values above the threshold define the signal subspace. 
Figure 4 shows the experimental guided mode phase velocities $c_{n}{ }^{\text {exp }}$ corresponding to the maxima of the Norm function [Eq. (9)] in the whole frequency-thickness bandwidth for the two probes, $1 \mathrm{MHz}(a)$ and $2 \mathrm{MHz}(b)$. The cut-off frequencies of each mode are indicated in the upper scale. Experimental values are in good agreement with the theoretical Lamb ones. At low frequencies, for $f e<1 \mathrm{MHz} . \mathrm{mm}$, only the $A_{0}$ mode is measured in both cases. At higher frequencies, most of the modes are evaluated. The $S_{2}$ backward wave, described for example in Ref. 33, is measured with the $1 \mathrm{MHz}$ probe $(a)$. These points are obtained using a negative phase velocity $c$. The cusp point for $f e=2.10 \mathrm{MHz} \cdot \mathrm{mm}$ and $c \approx 8,000 \mathrm{~m} . \mathrm{s}^{-1}$ is associated with the thin resonance peak on the singular values shown on Fig. 3. There is also a backward wave associated with the $S_{6}$ mode, for $f e=6.85 \mathrm{MHz} . \mathrm{mm}$, but the cusp point is outside the evaluation domain (i.e. $c>25,000 \mathrm{~m} \cdot \mathrm{s}^{-1}$ ). The phase velocities obtained with the spatio-temporal Fourier transform are marked with circles. The two methods are discussed in the next paragraph.

The two limits of the evaluation domain described in paragraph III.B are marked. The lowest limit corresponds to $\Delta c / c \approx 2 / N^{\mathrm{R}}=1 / 7 \approx 0.13$. The highest limit corresponds to $\Delta c / c \approx$ 2. Some resolution values $(1 / 4,1 / 2,1)$ are marked with dashed lines for reference. Following the resolution condition [Eq. (11)], two velocities are resolved when the ratio $\lambda / L^{\mathrm{R}}$ is less than the velocity difference $\Delta c / c$. In example $(a), A_{1}$ and $S_{2}$ modes are non-resolved for $f e$ about 2.2 MHz.mm, with $\Delta c / c \approx 0.3$. In example $(b), A_{3}$ and $S_{1}$ modes are also non resolved for $f e$ about 4.5 MHz.mm with $\Delta c / c \approx 0.2$. In these two cases the ratio $\lambda L^{\mathrm{R}}$ ranging from 0.25 to 0.5 is larger than the difference $\Delta c / c$. In other cases, the resolution condition is verified and the experimental phase velocities are resolved. 

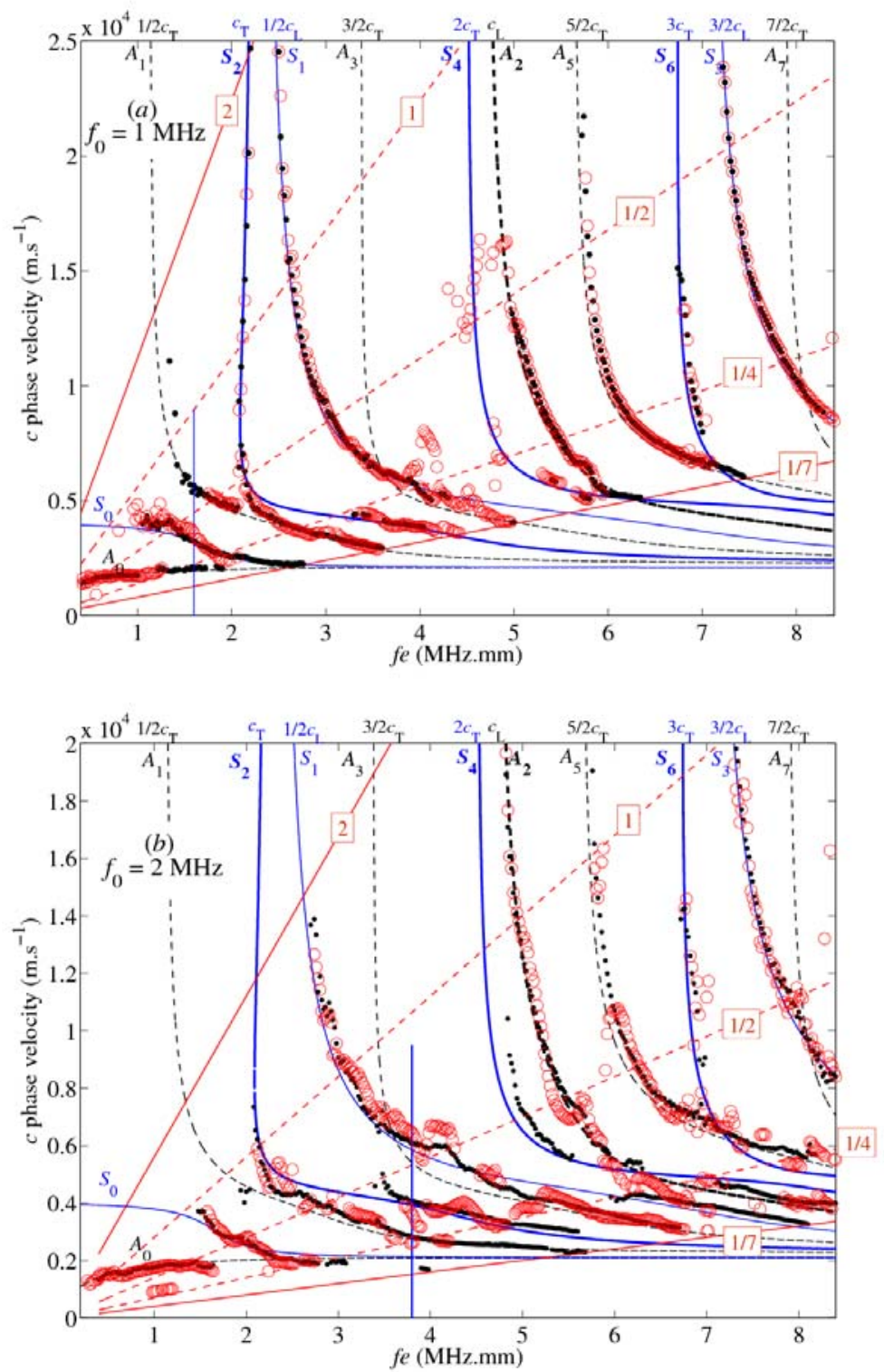

Fig. 4. (color online) Phase velocities for $2 \mathrm{~mm}$ thick copper plate, evaluated with probes of central frequency $f_{0}$ equal to $1 \mathrm{MHz}(a)$ and $2 \mathrm{MHz}(b)$. Theoretical Lamb values are shown in continuous (symmetric modes $S_{n}$ ) and dashed lines (anti-symmetric modes $A_{n}$ ). Experimental values, obtained with the maxima of the Norm function [Eq. (9)], are shown in dot. The phase velocities obtained with the spatio-temporal Fourier transform are marked with circles. The evaluation domain limit is shown with respect to Fig. 2. The ratios $\lambda L^{\mathrm{R}} 1 / 7,1 / 4,1 / 2,1$ and 2 are shown in thin dashed lines. The vertical lines show the domains discussed in Fig. 6. 
The values of the Lamb phase velocities depend on three physical parameters: the thickness $e$ and the transverse and longitudinal velocities, denoted $c_{\mathrm{T}}$ and $c_{\mathrm{L}}{ }^{32}$ It is possible to evaluate these parameters by searching the best fit between theoretical and experimental phase velocities, denoted $c_{n}^{\text {th }}$ and $c_{n}{ }^{\text {exp }}$, over the whole frequency bandwidth. ${ }^{35-37}$ The agreement is obtained here by minimizing the fitting error defined by the normalized sum of all individual fitting errors for each mode at each frequency

$$
\text { fitting error }\left(c_{\mathrm{L}}, c_{\mathrm{T}}, e\right)=100 \frac{1}{N^{\exp }} \sum_{f_{\min }}^{f_{\max }} \sum_{n=1}^{N^{\text {mode }}} \frac{\left|c_{n}^{\exp }(f)-c_{n}^{\text {th }}\left(c_{\mathrm{L}}, c_{\mathrm{T}}, e, f\right)\right|}{c_{n}^{\text {th }}\left(c_{\mathrm{L}}, c_{\mathrm{T}}, e, f\right)} \text {, }
$$

with $N^{\text {exp }}$ the number of experimental phase velocities, and $n$ the mode label, i.e. $A_{n}$ or $S_{n}$. The fitting errors are shown in Fig. 5 for the two probes as a function of $c_{\mathrm{L}}$ and $c_{\mathrm{T}}$ at a fixed thickness $e$ equal to $2 \mathrm{~mm}$. The fitting error values range from $5 \%$ to $30 \%$. Minima of the fitting errors yielded values of $c_{\mathrm{T}}=2250 \mathrm{~m} \cdot \mathrm{s}^{-1}$ and $c_{\mathrm{L}}=4700 \mathrm{~m} \cdot \mathrm{s}^{-1}$ for both probes. These values are consistent with the usual values for copper. 

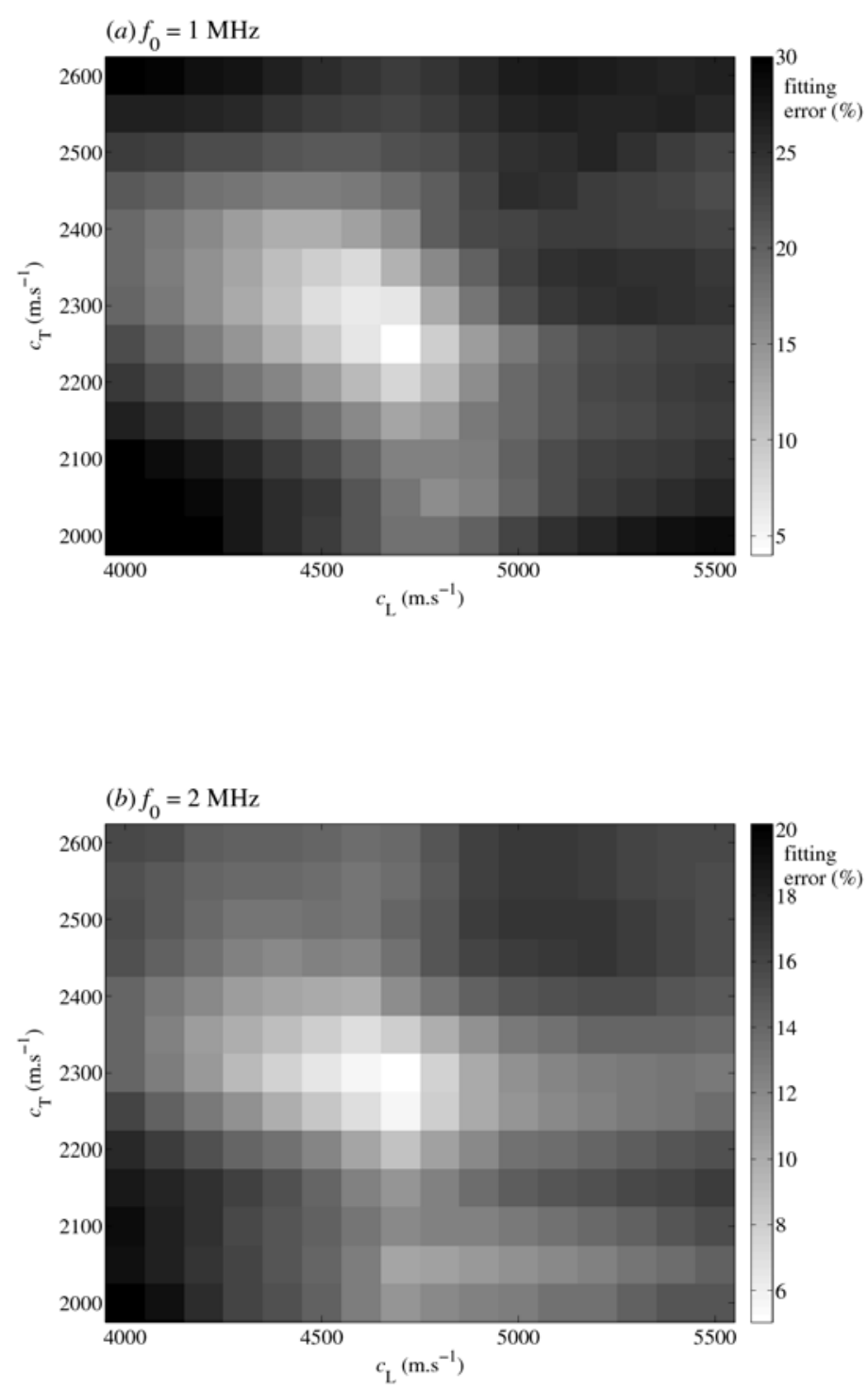

Fig. 5. Fitting error (\%) given by Eq. (12) as a function of $c_{\mathrm{L}}$ and $c_{\mathrm{T}}$ for a fixed thickness $e$, for a $2 \mathrm{~mm}$ thick copper plate, evaluated with probes of central frequency $f_{0}$ equal to $1 \mathrm{MHz}(a)$ and $2 \mathrm{MHz}(b)$, respectively. 


\section{Discussion, comparison with the spatio-temporal Fourier transform}

Figure 6 shows two examples of the evaluation of the guided mode phase velocities using the function $\operatorname{Norm}(f, c)$ and the spatio-temporal Fourier transform. Results are shown for a variable phase velocity $c$ at a fixed frequency : f.e $=1.6 \mathrm{MHz} . \mathrm{mm}$, with the $1 \mathrm{MHz}$ probe (a) and f.e $=3.8 \mathrm{MHz} . \mathrm{mm}$ with the $2 \mathrm{MHz}$ probe $(b)$. The domains are shown in Fig. 4 with vertical lines. The square of the scalar products $\left|\left\langle\mathbf{e}^{p w}(f, c) \mid \mathbf{U}_{n}\right\rangle\right|^{2}$ between the spatial plane wave and the three first singular vectors, $\mathbf{U}_{1}$ to $\mathbf{U}_{3}$, are shown with symbols $(\bullet,+$ and o). The width of the peaks corresponds to the resolution given by Eqs. (10) and (11).

The scalar products show how the singular vectors depend on the guided modes. In example (a), for $c$ around 3,200 m.s $\mathrm{s}^{-1}$, the first singular vector $\mathbf{U}_{1}$ is close to the guided mode $S_{0}$. For $c$ around 2,000 and 6,000 m.s $\mathrm{s}^{-1}$, the other singular vectors $\mathbf{U}_{2}$ and $\mathbf{U}_{3}$ are combination of the $A_{0}$ and $A_{1}$ modes. In example $(b)$, for $c$ around $6,500 \mathrm{~m} . \mathrm{s}^{-1}$, the first singular vector $\mathbf{U}_{1}$ is close to the guided mode $S_{1}$. The other singular vectors $\mathbf{U}_{2}$ to $\mathbf{U}_{3}$ are combination of the $A_{1}, S_{2}$ and $S_{1}$ modes, for $c$ around 2,500 4,000 and 6,500 m.s ${ }^{-1}$. Thus the guided mode phase velocities could be evaluated by searching the maxima of the moduli of the scalar products $\left|\left\langle\mathbf{e}^{p w}(f, c) \mid \mathbf{U}_{n}\right\rangle\right|^{2}$. However, the accuracy of that evaluation is improved if the different contributions are added in the function $\operatorname{Norm}(f, c)$ defined by Eqs. (8) and (9) and shown by a continuous line. The maxima of the Norm function, shown in these two examples, range from 0.6 to 0.95 : that is why the threshold $t_{2}$ described in paragraph II.C has been chosen equal to 0.5. Thus, the ability to determine guided mode phase velocities is improved by the use of the Norm function.

The two dimensional spatio-temporal Fourier transform is one of the usual methods used to determine the guided mode phase velocities. ${ }^{16}$ The scalar product $\left\langle\mathbf{e}^{p w}(f, c) \mid \mathbf{U}_{n}\right\rangle$ can be interpreted as the spatial Fourier transform of the reception singular vector $\mathbf{U}_{n}\left(x^{\mathrm{R}}\right)$, the 
spatial vector $k$ being equal to $2 \pi f / c$. The normalized spatio-temporal Fourier transform of the received signals on the array after the first emission, i.e. $\left|\left\langle\mathbf{e}^{p w}(f, c) \mid \mathbf{R}_{1}(f)\right\rangle\right|^{2}$, is shown by a dashed line. In example ( $a$ ), only one of the three present modes, $S_{0}$ is clearly resolved by the spatial Fourier transform. In example $(b)$, the three modes are resolved by the spatial Fourier transform, but the maxima ranges from 0.2 to 1 . As a comparison, the maxima of the Norm function are larger than 0.98 in this case. Thus the method proposed here appears to be more robust: the experimental guided modes phases velocities are better evaluated in the whole frequency domain as shown in Fig. 4. 
(a) $f . e=1.6 \mathrm{MHz} \cdot \mathrm{mm}\left(f_{0}=1 \mathrm{MHz}\right)$

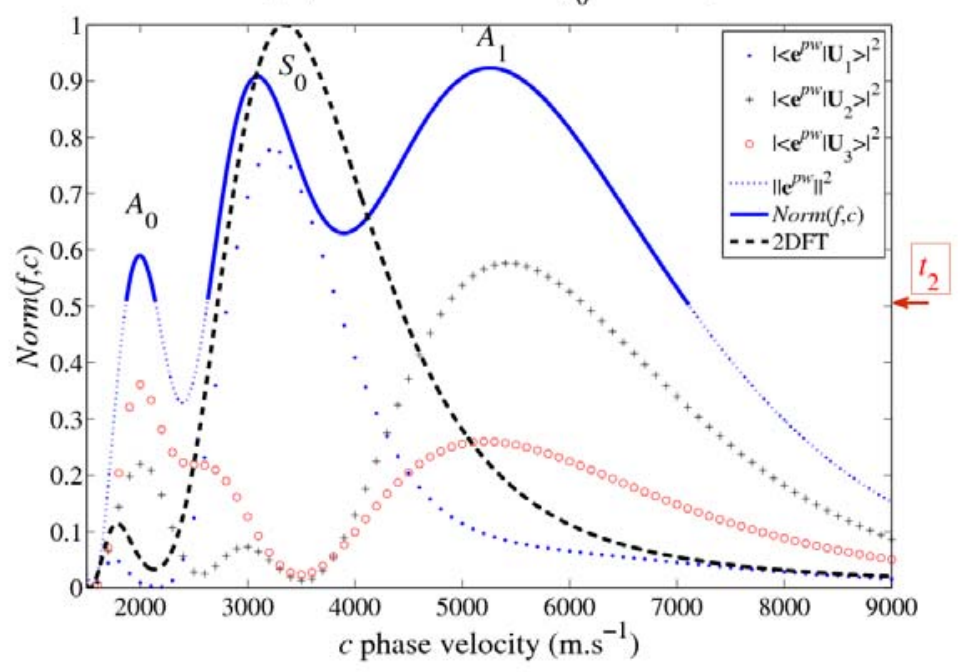

(b) $f . e=3.8 \mathrm{MHz} \cdot \mathrm{mm}\left(f_{0}=2 \mathrm{MHz}\right)$

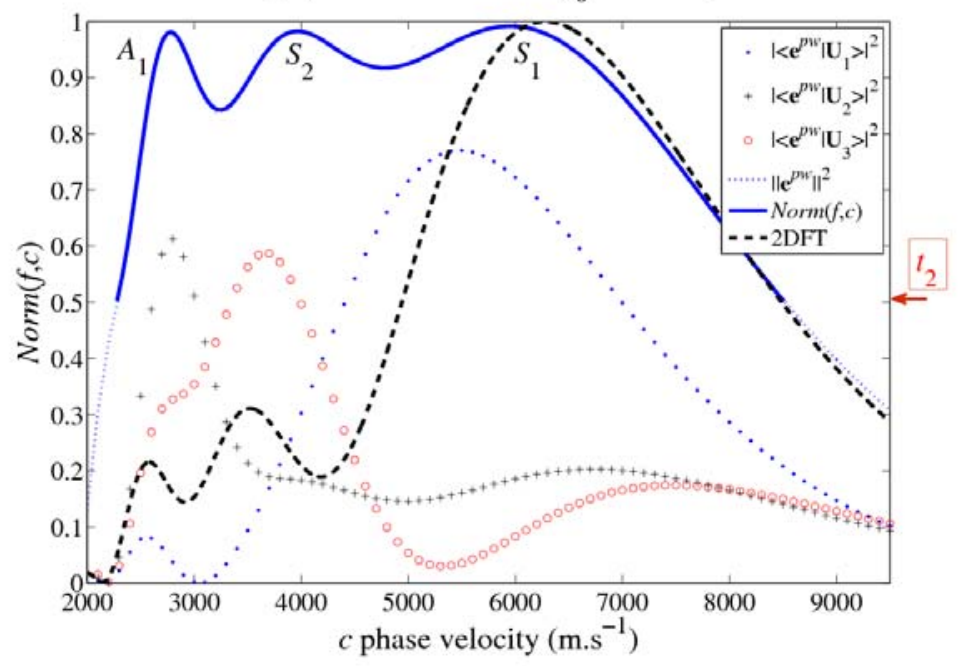

Fig. 6. (color online) Evaluation of the guided modes phase velocities at a fixed frequency: (a) $f e=1.6 \mathrm{MHz} . \mathrm{mm}$ (1 MHz probe) and (b) fe = 3.8 MHz.mm (2 MHz probe). The moduli of the first scalar products $\left|\left\langle\mathbf{e}^{p w} \mid \mathbf{U}_{n}\right\rangle\right|^{2}$ are shown with symbols $(\bullet+0)$. The norm of the plane wave on the basis of the signal subspace $\left\|\mathbf{e}^{p w}\right\|^{2}$ [Eq. (8)] is shown in thin dashed line. The phase velocities are given by the maxima of the function $\operatorname{Norm}(f, c)$ [Eq. (9)] above the threshold $t_{2}$ (shown with an arrow on the right side). The normalized spatio-temporal Fourier transform (2DFT) of the received signal after the first emission is shown in dashed thick line for comparison. 


\section{Conclusion}

An original method for evaluating without a priori the guided mode phase velocities, using a probe consisting of two separated emission and reception arrays in contact, is exposed in this paper. The phase velocities are obtained using the received signal subspace defined by the singular vectors of the experimental response matrix. The two main advantages of the method are presented: the signal-to-noise separation and the improvement of the ability to evaluate phase velocity compared to the spatio-temporal Fourier transform. To this end, two specific thresholds are described. Experimental results are in good agreement with the theoretical Lamb modes. These results hold promises in terms of evaluation of the wave guide structural and material properties using limited aperture array, andin terms of bone strength assessment. Further applications will concern in particular the evaluation of elastic properties of cortical bone. Attenuation non-isotropic medium, and variable thickness will be taken into account.

\section{Acknowledgments}

This work has been funded by the ANR TecSan. Authors would like to thank Dr. Claire Prada for the fruitful and helpful discussions. 


\section{References}

1 P. Laugier, "Instrumentation for in vivo assessment of bone strength," IEEE Trans. Ultrason. Ferroelec. Freq. Contr. 55, 1179-1196 (2008).

2 C. C. Gluer, “A new quality of bone ultrasound research,” IEEE Trans. Ultrason. Ferroelectr. Freq. Control. 55, 1524-1528 (2008).

3 P. Laugier, “Quantitative ultrasound of bone: looking ahead,” Joint Bone Spine 73, 125-128 (2006).

4 M. A. Krieg, R. Barkmann, S. Gonnelli, A. Stewart, D. C. Bauer, L. Del Rio Barquero, J. J. Kaufman, R. Lorenc, P. D. Miller, W. P. Olszynski, C. Poiana, A. M. Schott, E. M. Lewiecki, and D. Hans, “Quantitative ultrasound in the management of osteoporosis: the 2007 ISCD Official Positions,” J. Clin. Densitom. 11, 163-187 (2008).

5 P. Moilanen, P. H. F. Nicholson, V. Kilappa, S.L. Cheng, and J. Timonen, “Assessment of the cortical bone thickness using ultrasonic guided waves: Modelling and in vitro study,” Ultrasound Med. Biol. 33, 254-262 (2007).

6 F. Lefebvre, Y. Deblock, P. Campistron, D. Ahite, J. J. Fabre, “Development of a new ultrasonic technique for bone and biomaterials in vitro characterization,” J. Biomed. Mater. Res. 63, 441-446 (2002).

7 C. Desceliers, C. Soize, Q. Grimal, M. Talmant, and S. Naili, "Determination of the random anisotropic elasticity layer using transient wave propagation in a fluid-solid multilayer : model and experiments,” J. Acoust. Soc. Am. 125, 2027-2034 (2009).

8 E. Minnock, R. Cook, D. Collins, J. Tucker, and P. Zioupos, “Using Risk Factors and Quantitative Ultrasound to Identify Postmenopausal Caucasian Women at Risk of Osteoporosis,” Journal of Clinical Densitometry 11, 485-493 (2008). 
9 M. Maata, P. Moilanen, P. Nicholson, S. L. Cheng, J. Timonen, and T. Jamsa, “Correlation of Tibial Low-Frequency Ultrasound Velocity with Femoral Radiographic Measurements and BMD in Elderly Women,” Ultrasound Med. Biol. 35, 903-911 (2009).

10 A. Sarvazyan, A. Tatarinov, V. Egorov, S. Airapetian, V. Kurtenok, and C. J. Gatt Jr., “Application of the dual-frequency ultrasonometer for osteoporosis detection,” Ultrasonics 49, 331-337 (2009).

11 P. Moilanen, “Ultrasonic guided waves in bone,” IEEE Trans. Ultrason. Ferroelectr. Freq. Control 55, 1277-1286 (2008).

12 Barkmann R, Kantorovich E, Singal C, Hans D, Genant HK, Heller M, Gluer CC “A new method for quantitative ultrasound measurements at multiple skeletal sites - First Results of precision and fracture discrimination,” J. Clinical Densitometry 3, 1-7 (2000).

13 E. Bossy, M. Talmant, M. Defontaine, F. Patat, and P. Laugier, "Bidirectional axial transmission can improve accuracy and precision of ultrasonic velocity measurement in cortical bone: a validation on test materials,” IEEE Trans. Ultrason. Ferroelectr. Freq. Control. 51, 71-79 (2004).

14 E. Bossy, M. Talmant, P. Laugier, "Effect of bone cortical thickness on velocity measurements using ultrasonic axial transmission: A 2D simulation study,” J. Acoust. Soc. Am. 112, 297-307 (2002).

15 P. Moilanen, P. H. F. Nicholson, V. Kilappa, S. Cheng, and J. Timonen, "Measuring guided waves in long bones: Modeling and experiments in free and immersed plates,” Ultrasound Med. Biol. 32, 709-719 (2006).

D. Alleyne and P. Cawley, "A two-dimensional Fourier transform method for the measurement of propagating multimode signals,” J. Acoust. Soc. Am. 89, 1159-1168 (1991). 
17 M. Sasso, M. Talmant, G. Haiat, S. Naili, and P. Laugier, “Analysis of the most energetic late arrival in axially transmitted signals in cortical bone,” IEEE Trans. Ultrason. Ferroelectr. Freq. Control 56, 2463-2470 (2009). V. Vrabie, J. I. Mars, and J. L. Lacoume, "Singular value decomposition by means of independent component analysis,” Signal Processing 84, 645-652 (2004).

D. Ta, W. Wang, Y. Wang, L. H. Le, and Y. Zhou, "Measurement of the dispersion and attenuation of cylindrical ultrasonic guided waves in long bone,” Ultrasound in Medecine and Biology 35, 641-652 (2009). W. W. Sachse and Y. Pao, "On the determination of phase and group velocities of dispersive waves in solids,” J. Appl. Phys. 49, 4320-4327 (1978).

21 V. C. Protopappas, D. I. Fotiadis, K. N. Malizos, “Guided ultrasound wave propagation in intact and healing long bones,” Ultrasound Med. Biol. 32, 693-708 (2006).

W. H. Prosser, M. D. Seale, and B. T. Smith, “Time-frequency analysis of the dispersion of Lamb modes,” J. Acoust. Soc. Am. 105, 2669-2676 (1999).

D. Hans, L. Genton, S. Allaoua, C. Pichard, D. O. Slosman, "Hip fracture discrimination study - QUS of the radius and the calcaneum,” Journal of Clinical Densitometry 6, 163-172 (2003).

24 K. M. Knapp, G. M. Blake, T. D. Spector, and I. Fogelman, "Multisite quantitative ultrasound: Precision, age- and menopause-related changes, fracture discrimination, and T-score equivalence with dual-energy X-ray absorptiometry,” Osteoporosis Int. 12, 456-464 (2001).

M. Talmant, S. Kolta, Ch. Roux, D. Haguenauer, I. Vedel, B. Cassou, E. Bossy, P. Laugier, "In vivo performance evaluation of bi-directional ultrasonic axial transmission for cortical bone assessment,” Ultrasound Med. Biol. 35, 912-919 (2009). 
P. Moilanen, M. Talmant, V. Kilappa, P. Nicholson, S. Cheng, J. Timonen, and Pascal Laugier, "Modeling the impact of soft tissue on axial transmission measurements of ultrasonic guided waves in human radius,” J. Acoust. Soc. Am. 124, 2364 -2373 (2008). T. G. Kolda and B. W. Bader, “Tensor Decompositions and Applications,” SIAM Review 51, 455-500 (2009).

T. B. Neilsen, and E. K. Westwood, "Extraction of acoustic normal mode depth functions using vertical line array data,” J. Acoust. Soc. Am. 111, 748-756 (2002).

M. Cheney, "The linear sampling method and the MUSIC algorithm,” Inverse Problems 17, 591-595 (2001). C. Prada and M. Fink, "Separation of interfering acoustic scattered signals using the invariants of the time-reversal operator. Application to Lamb waves characterization,” J. Acoust. Soc. Am. 104, 801-807 (1998).

M. Davy, J. G. Minonzio, J. de Rosny, C. Prada, and M. Fink, “Influence of noise on subwavelength imaging of two close scatterers using time reversal method: Theory and Experiments,” Progress in Electromagnetic Research 98, 333-358 (2009). D. Royer, and E. Dieulesaint, "Elastics waves in solids 1 : Free and guided propagation,” Springer, Berlin, p. 317 (1999). C. Prada, D. Clorennec, and D. Royer, "Local vibration of an elastic plate and zero-group velocity Lamb modes,” J. Acoust. Soc. Am. 124, 203-212 (2008). I. Núñez, R. K. Ing, and C. Negreira, "Transfer and Green functions based on modal analysis for Lamb waves generation,” J. Acoust. Soc. Am. 107, 2370-2378 (2000). T. T. Wu and Y. H. Liu, "Inverse determinations of thickness and elastic properties of a bonding layer using laser-generated surface waves,” Ultrasonics 37, 23-30 (1999). W. Gao, C. Glorieux, and J. Thoen, "Laser ultrasonic study of Lamb waves: determination of the thickness and velocities of a thin plate,” Int. J. Eng. Sci. 41, 219-228 (2003). 
37 J. Vishnuvardhan, C. V. Krishnamurthy, and K. Balasubramaniama, "Blind inversion method using Lamb waves for the complete elastic property characterization of anisotropic plates,” J. Acoust. Soc. Am. Volume 125, 761-771 (2009). 
Fig. 1. Geometry of the problem: the emission and reception arrays are in contact with the metallic plate.

Fig. 2. The evaluation domain is shown outside the dashed domain. The resolution condition is given by Eq. (11): two velocities are resolved when their difference $\Delta c / c$ is larger than the ratio $\lambda / L^{\mathrm{R}}$ shown as linear function. The boundaries are shown on the experimental results (Fig. 4).

Fig. 3. (color online) Normalized singular values in $\mathrm{dB}$ scale versus frequency-thickness $f e$ (MHz.mm) for a $2 \mathrm{~mm}$ thick copper plate: the central frequency of the probe is equal to 1 $\mathrm{MHz}(a)$ and $2 \mathrm{MHz}(b)$. The number of experimental singular values (3 or 8) corresponds to the number of emitters. The signal-to-noise threshold $t_{1}$ is shown with a curved line. The singular values above the threshold define the signal subspace.

Fig. 4. (color online) Phase velocities for $2 \mathrm{~mm}$ thick copper plate, evaluated with probes of central frequency $f_{0}$ equal to $1 \mathrm{MHz}(a)$ and $2 \mathrm{MHz}(b)$. Theoretical Lamb values are shown in continuous (symmetric modes $S_{n}$ ) and dashed lines (anti-symmetric modes $A_{n}$ ). Experimental values, obtained with the maxima of the Norm function [Eq. (9)], are shown in dot. The phase velocities obtained with the spatio-temporal Fourier transform are marked with circles. The evaluation domain limit is shown with respect to Fig. 2 . The ratios $\lambda L^{\mathrm{R}} 1 / 7,1 / 4,1 / 2,1$ and 2 are shown in thin dashed lines. The vertical lines show the domains discussed in Fig. 6. 
Fig. 5. Fitting error (\%) given by Eq. (12) as a function of $c_{\mathrm{L}}$ and $c_{\mathrm{T}}$ for a fixed thickness $e$, for a $2 \mathrm{~mm}$-thick copper plate, evaluated with probes of central frequency $f_{0}$ equal to $1 \mathrm{MHz}(a)$ and $2 \mathrm{MHz}(b)$, respectively.

Fig. 6. (color online) Evaluation of the guided modes phase velocities at a fixed frequency: (a) $f e=1.6 \mathrm{MHz} . \mathrm{mm}$ (1 MHz probe) and (b) fe = 3.8 MHz.mm (2 MHz probe). The moduli of the first scalar products $\left|\left\langle\mathbf{e}^{p w} \mid \mathbf{U}_{n}\right\rangle\right|^{2}$ are shown with symbols $(\bullet+0)$. The norm of the plane wave on the basis of the signal subspace $\left\|\mathbf{e}^{p w}\right\|^{2}$ [Eq. (8)] is shown in thin dashed line. The phase velocities are given by the maxima of the function $\operatorname{Norm}(f, c)$ [Eq. (9)] above the threshold $t_{2}$ (shown with an arrow on the right side). The normalized spatio-temporal Fourier transform (2DFT) of the received signal after the first emission is shown in dashed thick line for comparison. 


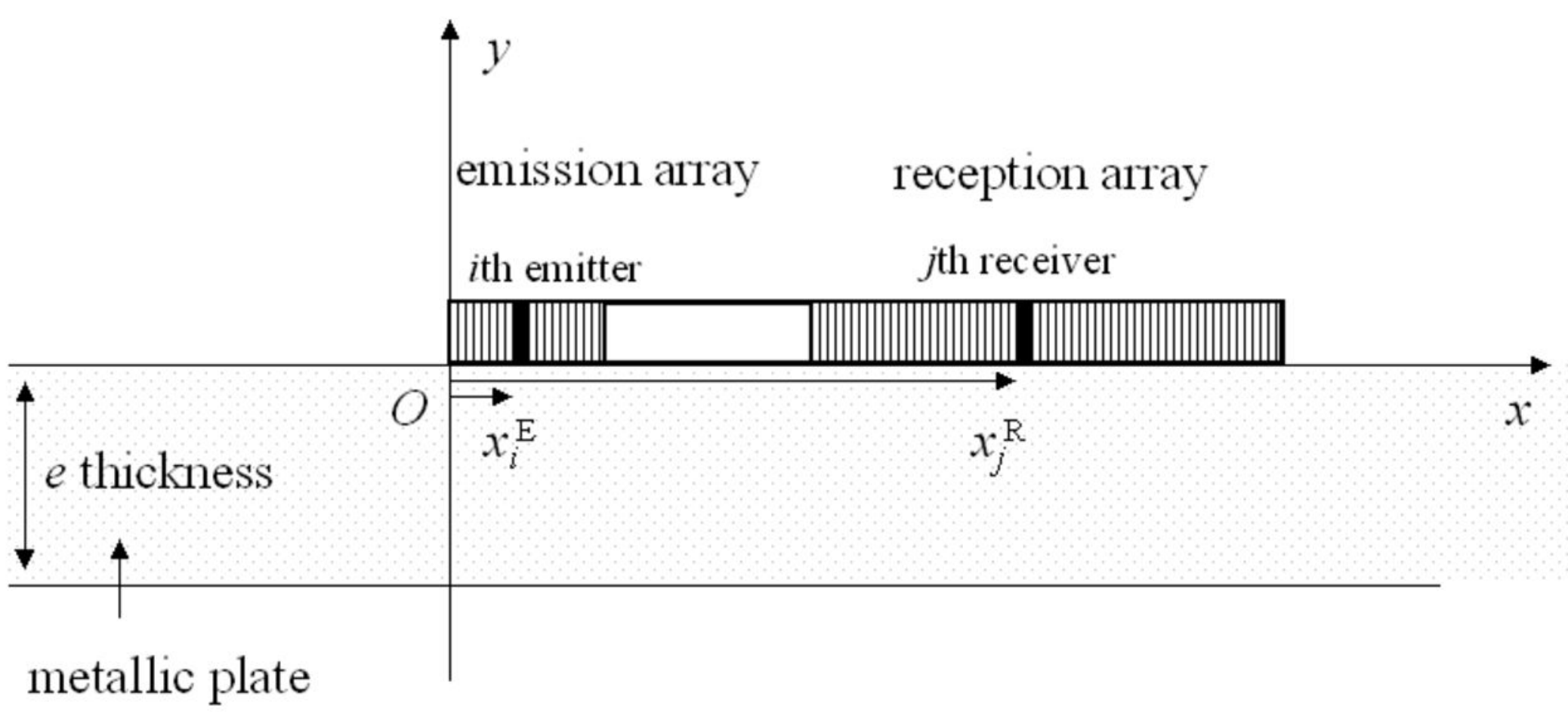




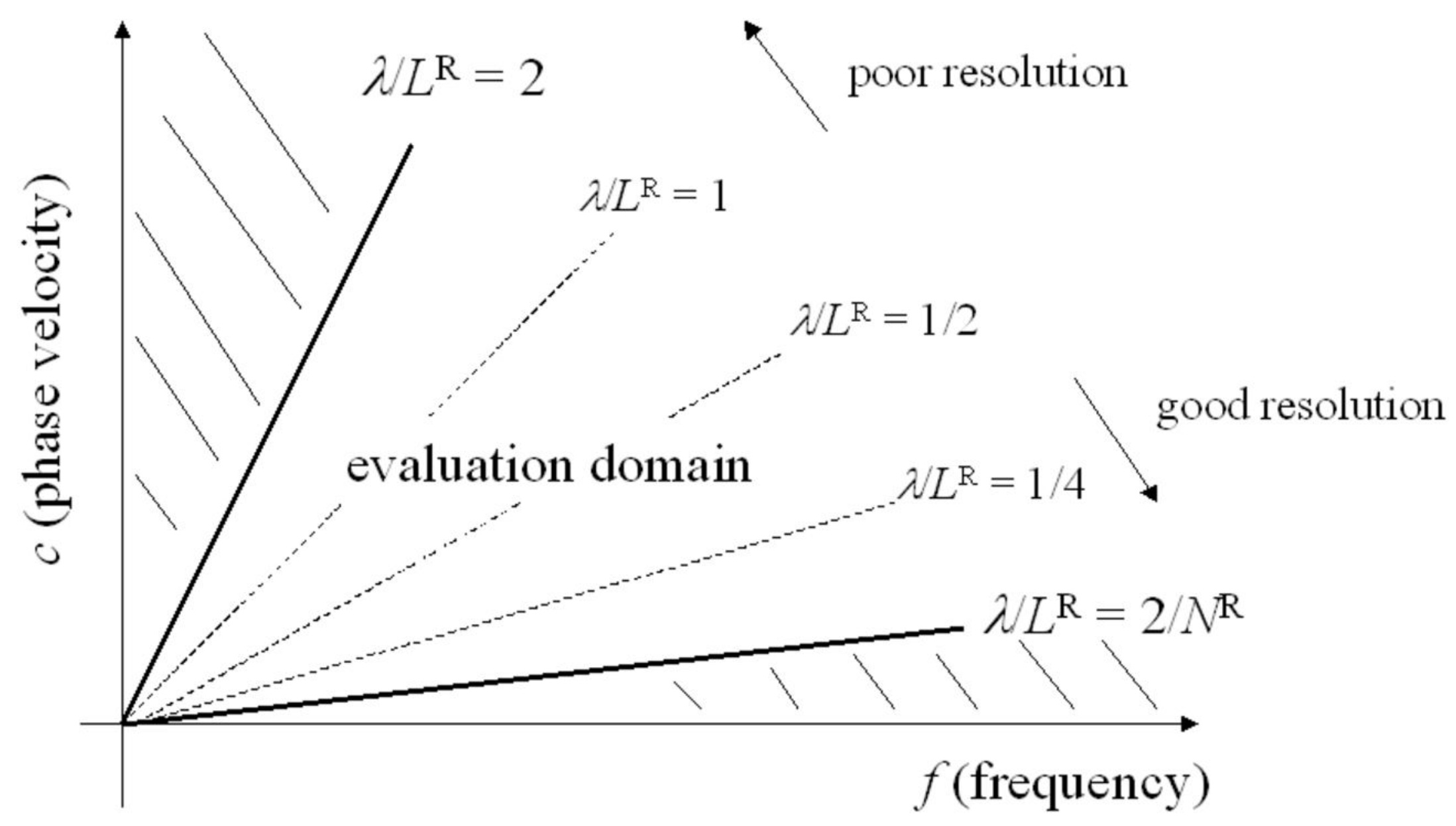




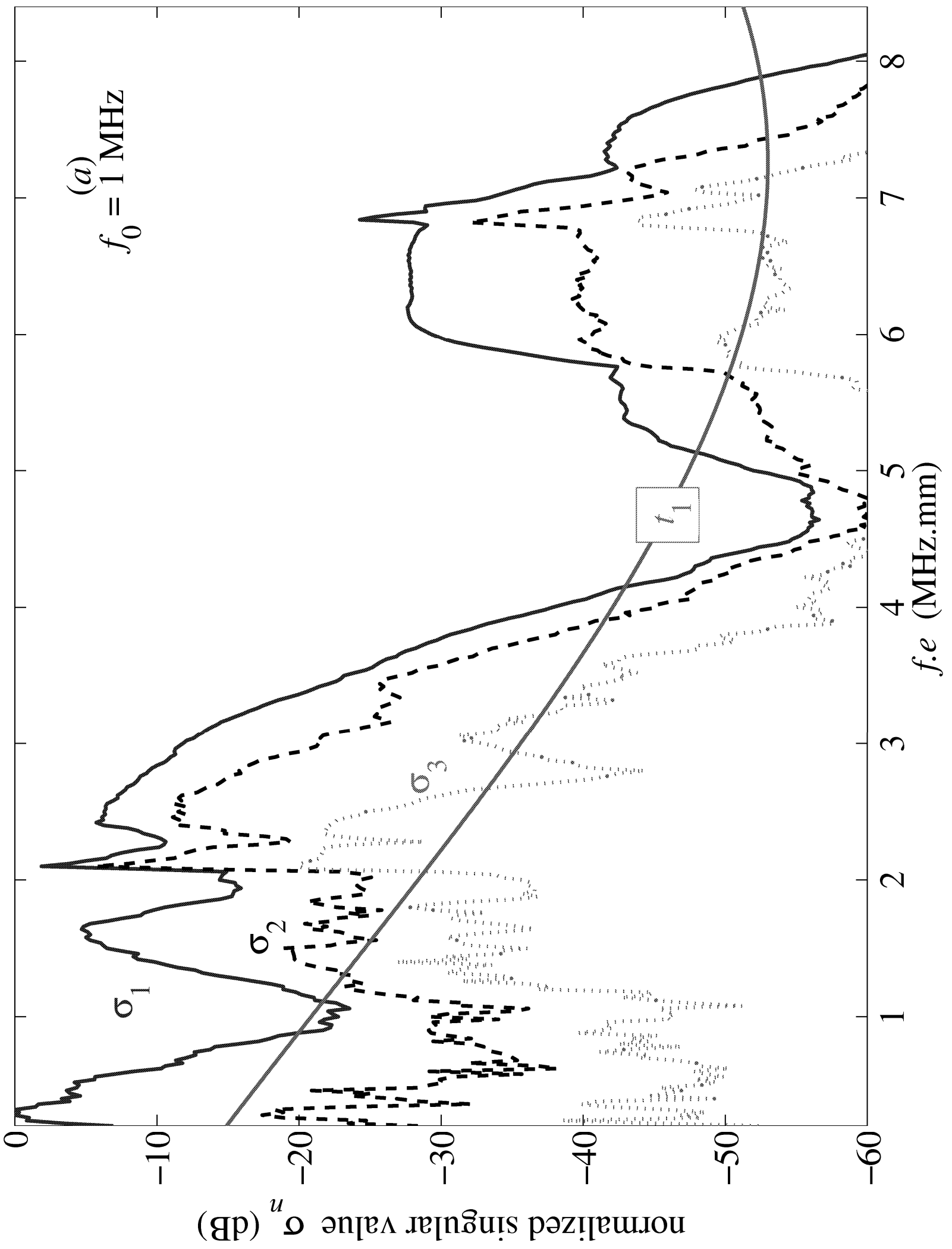




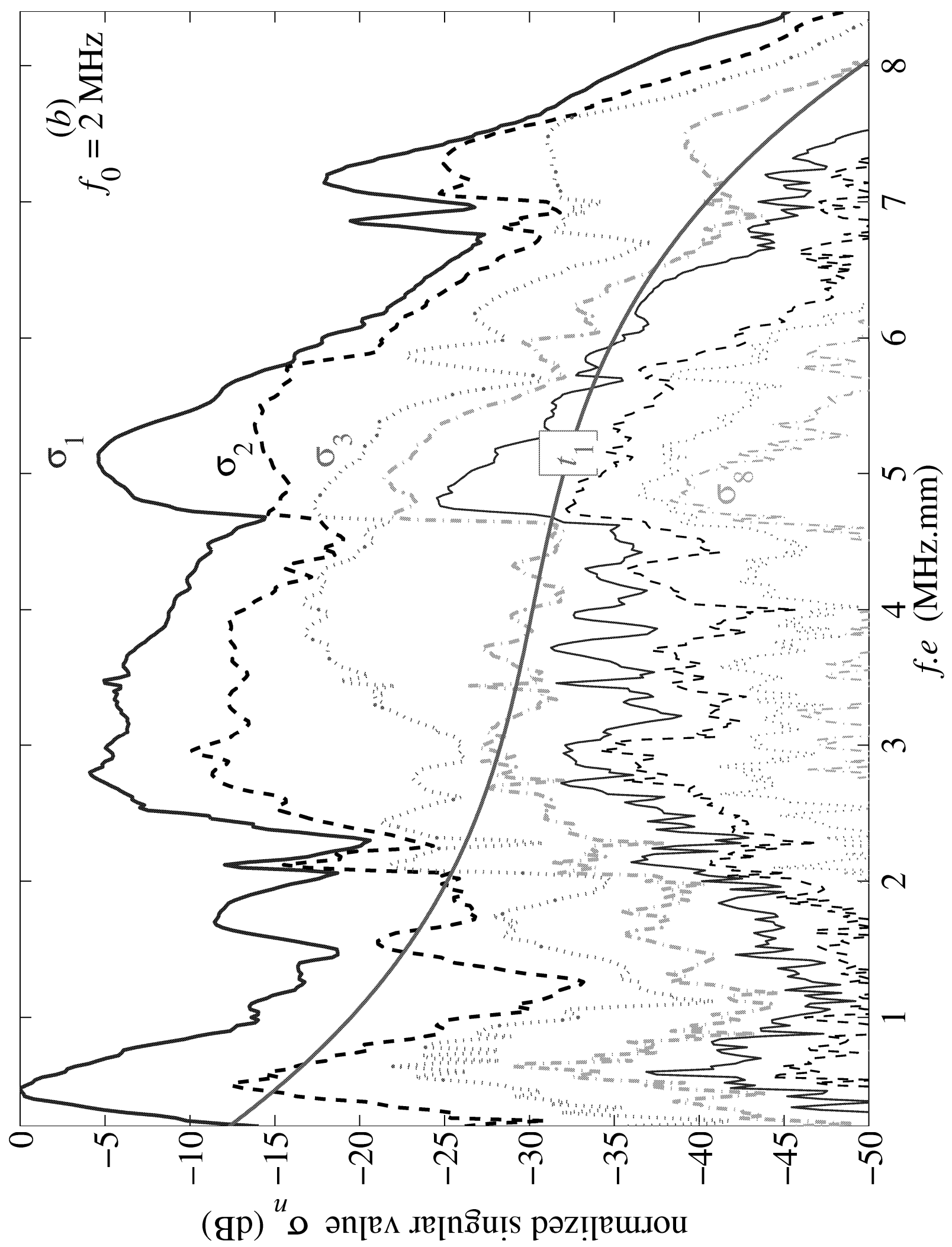




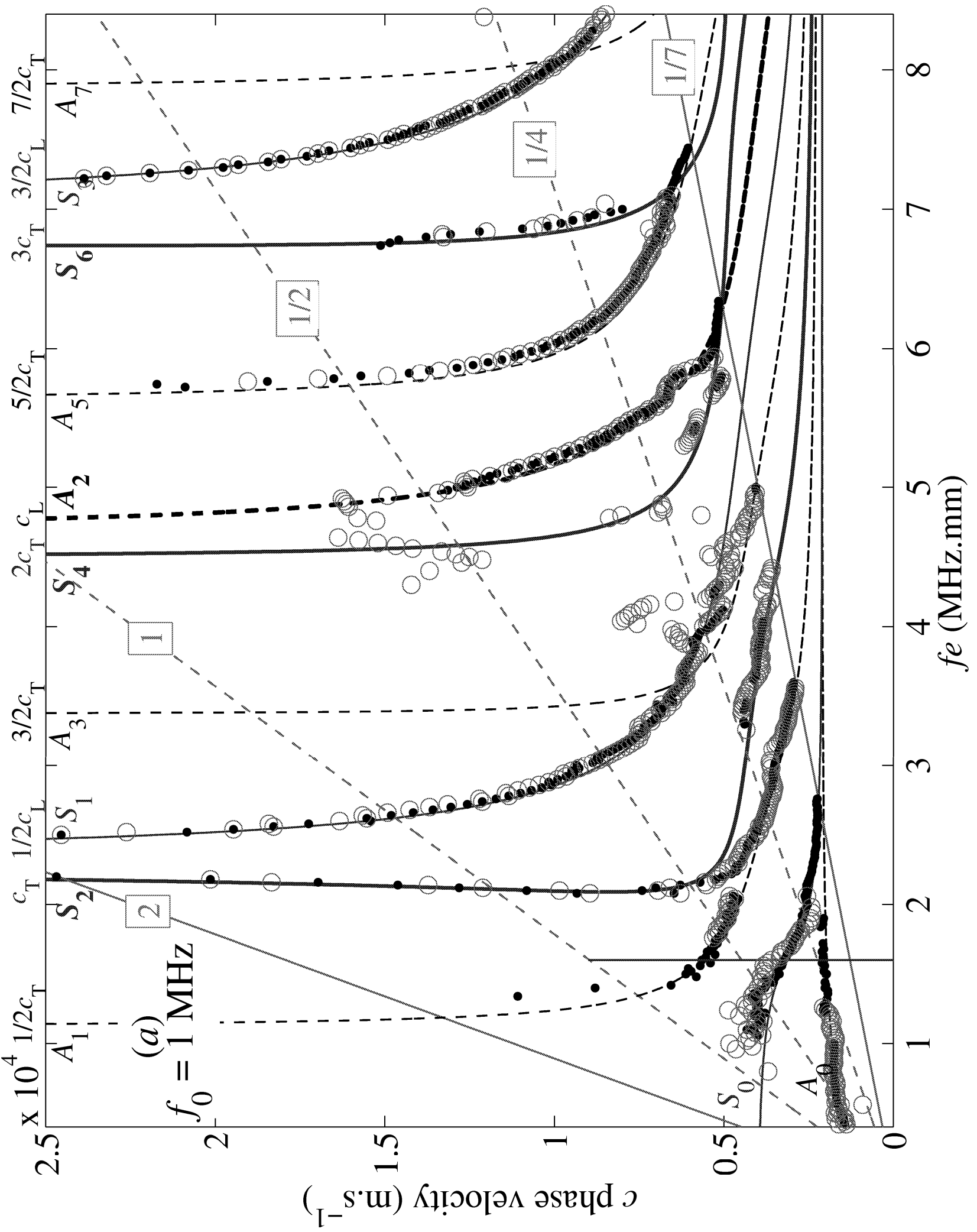




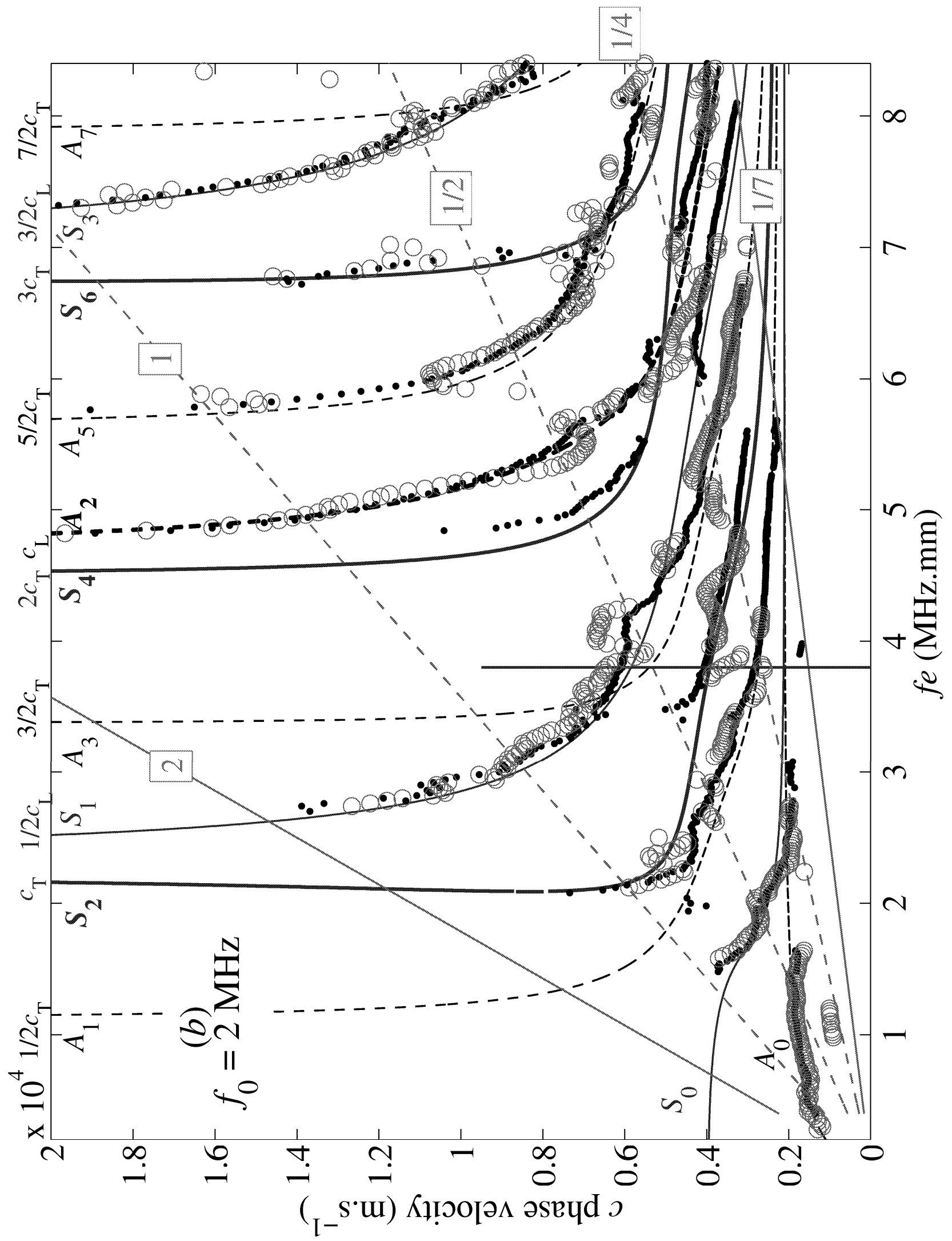




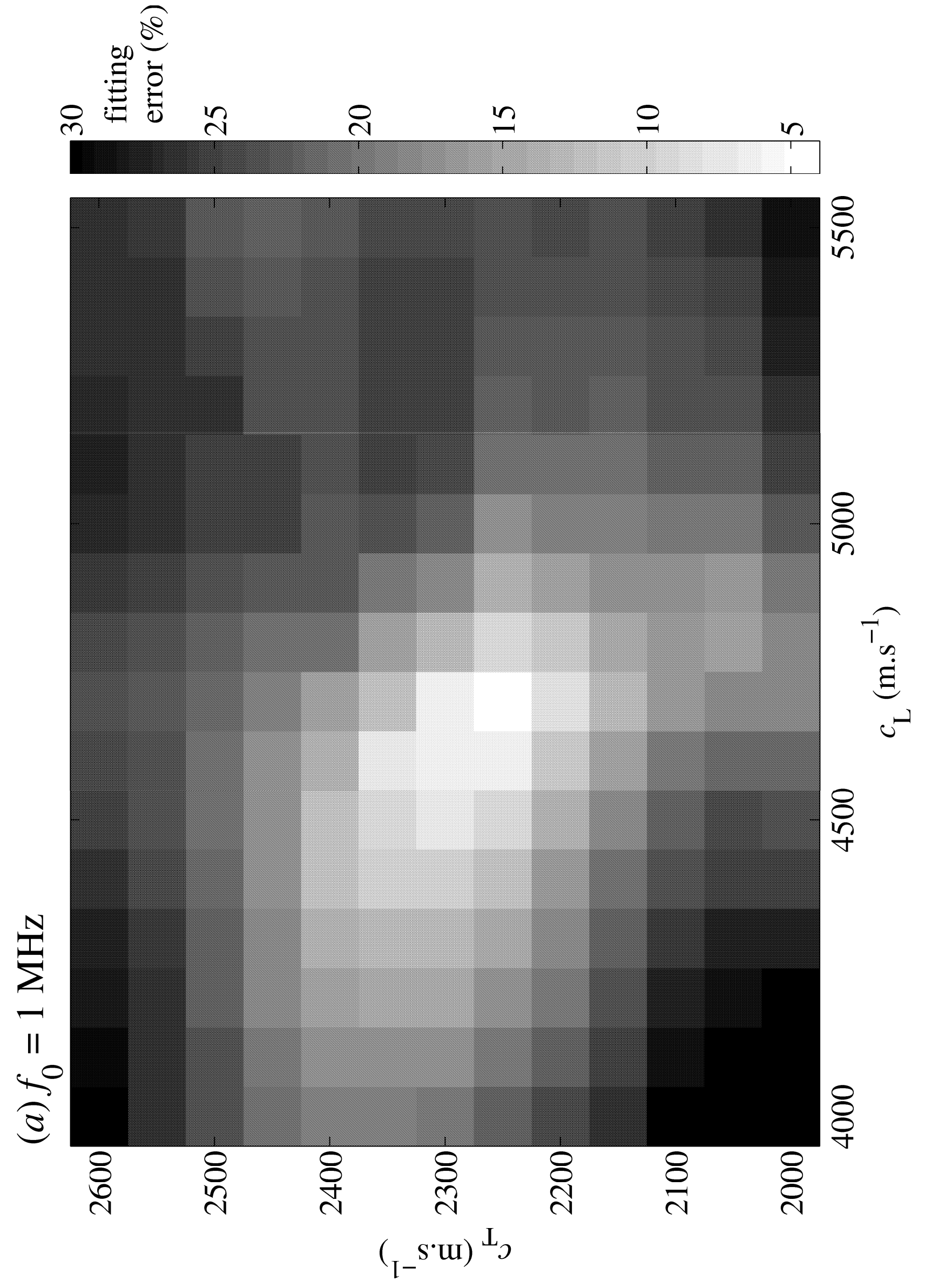




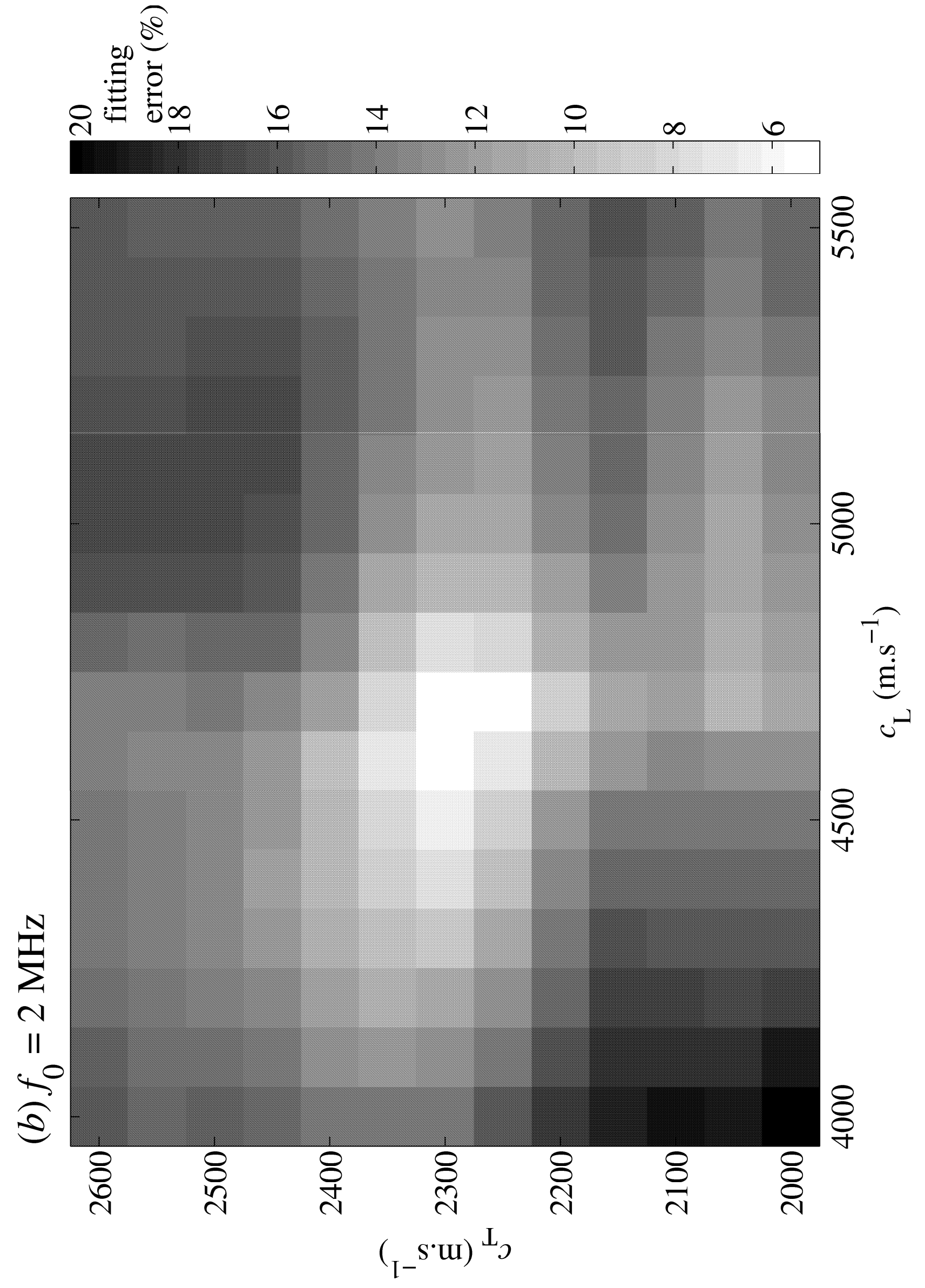




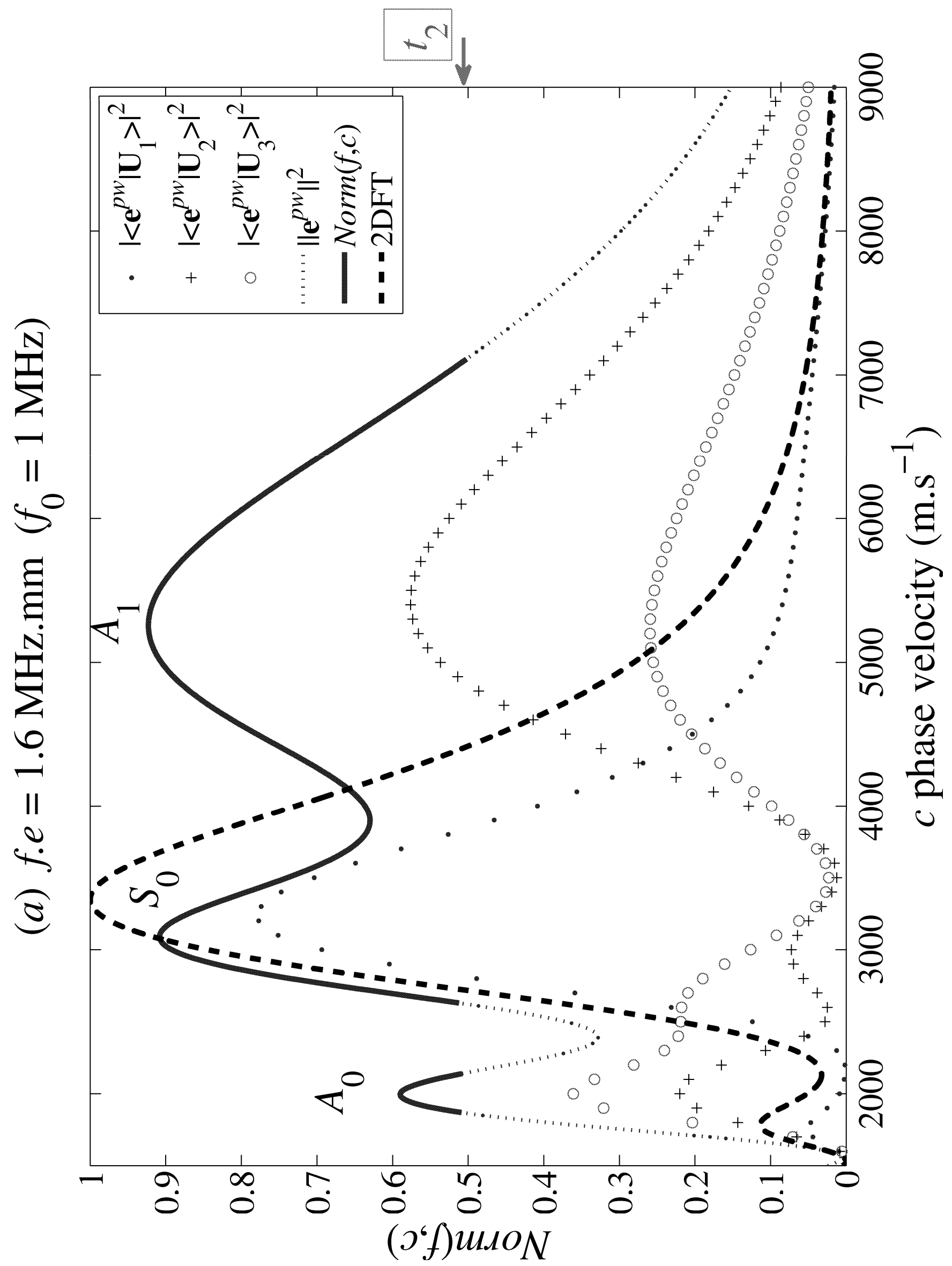




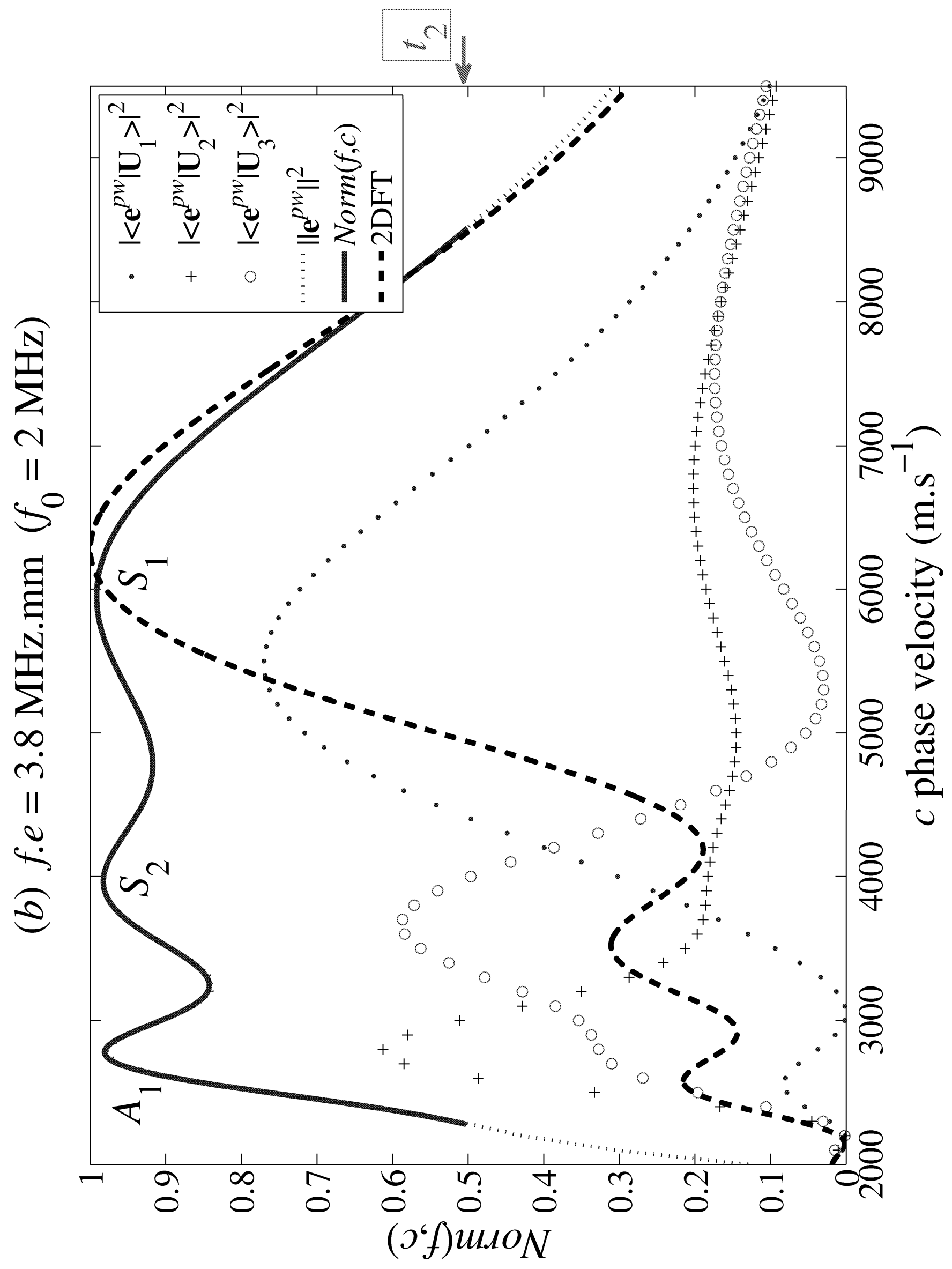

